\title{
MEASURING ROCKET ENGINE TEMPERATURES WITH HYDROGEN RAMAN SPECTROSCOPY
}

\author{
Joseph A. Wehrmeyer \\ Mechanical Engineering Department, Vanderbilt University \\ Box 1592 Station B, Nashville, TN 37235 \\ Robin J. Osborne \\ ERC. Inc. \\ NASA-Marshall Space Flight Center, Huntsville, AL 35812 \\ Huu P. Trinh \\ Space Transportation Directorate \\ NASA-Marshall Space Flight Center, Huntsville, AL 35812
}

\begin{abstract}
Optically accessible, high pressure, hot fire test articles are available at NASA Marshall for use in development of advanced rocket engine propellant injectors. Single laser-pulse UV Raman spectroscopy has been used, in the past, in these devices for analysis of high pressure $\mathrm{H}_{2-}$ and $\mathrm{CH}_{4}$-fueled combustion, but relies on an independent pressure measurement in order to provide temperature information. A variation of UV Raman (High Resolution Hydrogen Raman Spectroscopy) is under development and will allow temperature measurement without the need for an independent pressure measurement, useful for flows where local pressure may not be accurately known. The technique involves the use of a spectrometer with good spectral resolution, requiring a small entrance slit for the spectrometer. The $\mathrm{H}_{2}$ Raman spectrum, when created by a narrow linewidth laser source and obtained from a good spectral resolution spectrograph, has a spectral shape related to temperature. By best-fit matching an experimental spectrum to theoretical spectra at various temperatures, a temperature measurement is obtained. The spectral model accounts for collisional narrowing, collisional broadening, Doppler broadening, and collisional line shifting of each Raman line making up the $\mathrm{H}_{2}$ Stokes vibrational Q-branch spectrum. At pressures from atmospheric up to those associated with advanced preburner components (5500 psia), collisional broadening though present does not cause significant overlap of the Raman lines, allowing high resolution $\mathrm{H}_{2}$ Raman to be used for temperature measurements in plumes and in high pressure test articles. Experimental demonstrations of the technique are performed for rich $\mathrm{H}_{2}$-air flames at atmospheric pressure and for high pressure, $300 \mathrm{~K} \mathrm{H}_{2}$-He mixtures. Spectrometer imaging quality is identified as being critical for successful implementation of technique.
\end{abstract}

\section{INTRODUCTION}

Laser-based combustion diagnostics, such as single-pulse UV Raman spectroscopy (Wehrmeyer et al. 2001) and visible Raman spectroscopy (Yeralan et al. 2001), have been successfully applied to optically-accessible rocket-like test articles. If an independent pressure measurement is available, Raman major species concentration measurements can also provide a temperature 
measurement. However it is desirable to obtain a Raman-derived temperature measurement without the need for simultaneous pressure measurement, especially when chamber pressure may vary spatially. This report describes Raman temperature measurements obtained by exploiting the variation in shape of the $\mathrm{H}_{2}$ Raman spectrum. Hydrogen is advantageous since it is ubiquitous in $\mathrm{H}_{2}-\mathrm{O}_{2}$ systems and its Raman spectrum is simpler than for other diatomics. However the influence of high pressure on the $\mathrm{H}_{2}$ Raman spectrum must be investigated. At moderate pressures, well below those of rocket engines, the Raman spectra of $\mathrm{O}_{2}$ and $\mathrm{N}_{2}$ are known to become featureless due to collisional broadening (Eckbreth 1988).

\section{THEORETICAL H2 RAMAN SPECTRA：WAVELENGTH, LINE STRENGTH, AND LINEWIDTH}

Hydrogen is a centrifugally-stretched rotor with rotational energy, $E_{r}$, given by (Eckbreth 1988):

$$
E_{,}=h c\left[B J(J+1)-D J^{2}(J+1)^{2}+\ldots\right]
$$

where $h$ is Planck's constant $\left(6.6 \times 10^{-34}\right.$ joule-sec $), c$ is speed of light $\left(3 \times 10^{10} \mathrm{~cm} / \mathrm{sec}\right), B$ is the rotational term value, in $\mathrm{cm}^{-1}$ (wavenumbers), $D$ is a centrifugal stretch correction term value, and $J$ is rotational quantum number $\left(0,1\right.$, etc.) Assuming $\mathrm{H}_{2}$ is also an anharmonic oscillator, its vibrational energy can be modeled as a function of vibrational quantum number, $v(0,1$, etc. $)$, by:

$$
E_{1}=h c\left[\omega_{c}\left(v+\frac{1}{2}\right)-\omega_{e^{\prime}} x_{c}\left(v+\frac{1}{2}\right)^{2}+\ldots\right]
$$

where $\omega_{e}$ is the fundamental vibrational term value and $x_{c}$ is an anharmonicity correction factor. Both $B$ and $D$ are functions of $v$ through vibration-rotation interaction and are given $v$ subscripts:

$$
B_{v}=B_{c}-a_{c}\left(v+\frac{1}{2}\right)+\ldots \quad D_{v}=D_{c}+\beta_{c}\left(v+\frac{1}{2}\right)+\ldots
$$

where $B_{\ell}, D_{\ell}, \alpha_{\ell}$, and $\beta_{\iota}$, are constants. Values are in Jennings et al. (1985) and Weber (1973).

Spontaneous Raman scattering is the inelastic scattering of light by a molecule. For Stokes vibrational Q-branch Raman transitions, the molecular internal energy change obeys $\Delta v=+1, \Delta J$ $=0$. The increase in the scattering molecule's final energy, $E^{\prime}$ from its initial energy, $E^{\prime \prime}$. is:

$$
E^{\prime}-E^{\prime \prime}=h c\left[\omega_{\iota^{\prime}}-2 \omega_{e} x_{\iota^{\prime}}\left(v^{\prime \prime}+1\right)-\alpha_{\iota^{\prime}} J(J+1)-\beta_{e^{\prime}} J^{2}(J+1)^{2}+\ldots\right]
$$

where $v^{\prime \prime}$ is $v$ for the initial energy state. Equation 5 gives the energy lost by the interacting photons, and since their wavelengths are related to their energies, the wavelength of the Raman scattered light, $\lambda_{R}$, will depend upon the incident photon's wavelength, $\lambda_{I}$, and $v^{\prime \prime}$ and $J$ through:

$$
\lambda_{k}=\left\{\lambda_{t}{ }^{-1}-\left[\omega_{c}-2 \omega_{t} x_{t^{\prime}}\left(v^{\prime \prime}+1\right)-\alpha_{t} J(J+1)-\beta_{c} J^{2}(J+1)^{2}+\ldots\right]\right\}^{-1}
$$


Since $\omega_{c} \gg \omega_{c^{\prime}} x_{c^{\prime}} \gg \alpha_{c^{\prime}} \gg \beta_{e}, \omega_{\epsilon}$ approximately determines the $\lambda_{R}$ 's; next in importance is $\omega_{e} x_{c}$, which groups together Raman lines according to $v^{\prime \prime}$; and within each $v^{\prime \prime}$ group the $J$ value determines a line's exact $\lambda_{R}$. Actually, $E^{\prime}-E^{\prime \prime}$ also depends upon temperature, $T$, and density, $\rho$, through vibrational perturbations, which can either reduce or increase $E^{\prime}-E^{\prime \prime}$ through the relative importance of short-range repulsive forces compared to long-range attractive forces (May et al. 1964). A correction term, $\Delta E / h c$, can be added to Eq. 6 for the $\rho^{-}, T-, J_{-}$, and collision partnerdependent shift of $\lambda_{k}$. Values for $\Delta E / h c$ are available for $\mathrm{H}_{2}$ in $\mathrm{H}_{2}$ (May et al. 1964), $\mathrm{H}_{2}$ in $\mathrm{H}_{2}$, Ar, $\mathrm{He}, \mathrm{N}_{2}$, or $\mathrm{CH}_{4}$ (Lallemand and Simova 1968), and $\mathrm{H}_{2}$ in $\mathrm{N}_{2}$ (Sinclair et al. 1996).

Each Raman line's intensity is related to its $v^{\prime \prime}, J$ level population and its transition probability (cross section). Calculated cross sections, $\sigma_{v}^{\prime \prime}=\left(0, J\right.$. for $\mathrm{H}_{2}$ 's $v^{\prime \prime}=0, J=0$ to $J=3$ levels are available (Ford and Browne 1973) and higher vibrational level cross sections scale as $\left(v^{\prime \prime}+1\right)$ (Placzek 1932). Assuming a Boltzmann distribution, each Raman line's intensity, $I_{R}$, is related to $T$ by:

$$
I_{R} \propto \sigma_{v^{*}=0 . J}\left(v^{\prime \prime}+1\right) Q^{-1} g^{\prime \prime}(2 J+1) \exp \left(-E^{\prime \prime} / k T\right)
$$

where $Q$ is the $T$-dependent ro-vib-nuc partition function, $k$ is Boltzmann's constant ( $1.38 \times 10^{-23}$ joule/K), and $\mathrm{g}^{\prime \prime}$ is relative degeneracy for ro-nuclear spin coupling (for $\mathrm{H}_{2}: 1$ odd $J, 3$ even $J$ ).

Figures 1 and 2 show Stokes vibrational Q-branch Raman spectra (for $248.623 \mathrm{~nm}$ excitation) at two $T s, 700 \mathrm{~K}$ and $3400 \mathrm{~K}$, and use the lineshift data of Sinclair et al. (1996) for $\mathrm{H}_{2}$ diluted in $\mathrm{N}_{2}$. At $700 \mathrm{~K}$ only the first seven rotational levels for $v^{\prime \prime}=0$ are significantly populated. At 3400 $\mathrm{K}$ more rotational levels in the ground and excited vibrational states become populated due to the $T$ dependence of the Boltzmann fraction factor in Eq. 7.

A Raman line's width, $\Gamma$ (in $\mathrm{cm}^{-1}$ ), is not zero, as in Figs. 1 and 2, but is finite and depends upon molecular velocities and collisions, hence is a function of $T, \rho$, collision partner, and collection geometry. At low $\rho$ a Doppler-broadened gaussian lineshape occurs with a linewidth. $\Gamma_{D i}$, dependent upon angle, $\theta$, between incident and scattered photon directions (Weber 1973):

$$
\Gamma_{D \prime \eta, p^{\prime}}=2[2 \ln (2) k T / m]^{1 / 2}\left[4\left(\lambda_{L}^{-2}+\lambda_{l}^{-1} \frac{E^{\prime \prime}-E^{\prime}}{h c}\right) \sin ^{2} \theta / 2+\left(\frac{E^{\prime \prime}-E^{\prime}}{h c}\right)^{2}\right]^{1 / 2}
$$

where $m$ is the molecular mass. Thus $\Gamma_{D_{1}}$ is a minimum for forward scattering $\left(\theta=0^{\circ}\right)$ and a maximum for backward scattering $\left(\theta=180^{\circ}\right)$ and intermediate for $\theta=90^{\circ}$.

Because of the Uncertainty Principle, a Doppler shifted photon gives velocity information for a molecule that is averaged across a distance it travels, given by $\lambda_{R} / 2 \pi$ (Murray and Javan 1972). For increasing number of velocity changing collisions experienced through this travel length, the molecule's average velocity and Doppler shift will approach zero. This Dicke (or collisional) narrowing and can be thought of as a viscous drag, exerted on the scattered photon, that increases with $\rho$. If the mean free path $<\lambda_{R} / 2 \pi$, a Dicke-narrowed linewidth $\left(\Gamma_{D i c k e}\right)$ can be modeled as: 


$$
\Gamma_{D_{i t k c}}=\left[4 \pi D_{0} / c \rho\right]\left[4\left(\lambda_{l}^{-2}+\lambda_{l}^{-1} \frac{E^{\prime \prime}-E^{\prime}}{h c}\right) \sin ^{2} \theta / 2+\left(\frac{E^{\prime \prime}-E^{\prime}}{h c}\right)^{2}\right]^{1 / 2}
$$

where $D_{\vartheta}$, in $\mathrm{cm}^{2} /(\mathrm{sec}$ amagat $)$, is an "optical" diffusion coefficient $\approx 1.13 \times$ molecular diffusion coefficient (Rahn et al. 1991; Bergmann and Stricker 1995). One amagat $=\rho$ at $1 \mathrm{~atm}, 273 \mathrm{~K}$. For large $\rho$ collisional broadening occurs due to collisions that terminate, rather than perturb, the scattering process. The resulting finite-lived wavetrain has a frequency, or wavenumber. spread inversely proportional to its duration. The collisionally-broadened linewidth can be modeled as:

$$
\Gamma_{\text {cinll. Broutl }}=2 \gamma_{j}^{0} \rho
$$

where $\gamma_{J}$ is a broadening coefficient dependent on $J$, collision partner, and $T$, and $\gamma^{\prime}$, has been modeled by Hussong et al. (2000) for $\mathrm{H}_{2}-\mathrm{H}_{2}$ and $\mathrm{H}_{2}-\mathrm{N}_{2}$ collisions. Thus $\Gamma$ is composed of a combination of three terms, each term dominating within a $\rho$ range: $\Gamma=\Gamma_{\text {Domp }}$ at low $\rho, \Gamma=\Gamma_{\text {Dicke }}$ at medium $\rho$, and $\Gamma=\Gamma_{\text {Coll }}$ Broul at high $\rho$. Figure 3 shows the first two rotational lines of the $\mathrm{H}_{2}$ Stokes vibrational Q-branch spectrum at $300 \mathrm{~K}$, for dilute $\mathrm{H}_{2}$ in $\mathrm{N}_{2}$. At 1 atm $(0.911$ amagat) and $10 \mathrm{~atm}$ (9.14 amagat) Dicke narrowing dominates, with $\Gamma$ less at $10 \mathrm{~atm}$ than $1 \mathrm{~atm}$. At $100 \mathrm{~atm}$ (91.9 amagat from Redlich-Kwong data) collisional broadening dominates, but the lines are fully resolved and remain so even at $370 \mathrm{~atm}$, a pressure expected in advanced preburners.

\section{EXPERIMENTAL RAMAN SPECTRUM}

Using the experimental system, shown in Fig. 4, of Wehrmeyer et al. (2001), a demonstration of high resolution $\mathrm{H}_{2}$ Raman spectroscopy is performed in an atmospheric pressure $\mathrm{H}_{2}$-air flame. A tunable krypton-fluoride excimer laser with a laser linewidth, $\Delta \lambda_{L}$, of $0.003 \mathrm{~nm}$, is used along with an $0.3 \mathrm{~m}$ imaging spectrograph with an imaging resolution, $\Delta \lambda_{s}$, of $0.2 \mathrm{~nm}$. Both $\Delta \lambda_{S}$ and $\Delta \lambda_{l}$ cause the measured linewidth of each Raman line, $\Delta \lambda_{R}$, to be greater than $\Gamma / \lambda_{R}{ }^{2}$, (the "natural" linewidth expressed in wavelength units rather than wavenumbers). Assuming gaussian lineshapes, $\Delta \lambda_{S}$ and $\Delta \lambda_{L}$ can be convoluted together to form a gaussian "instrumental" lineshape with a FWHM of $\left(\Delta \lambda_{S}{ }^{2}+\Delta \lambda_{L}{ }^{2}\right)^{1 / 2}$. In addition the $100 \mu \mathrm{m}$ spectrograph slit also broadens the instrumental linewidth through its further convolution with a rectangular lineshape of $0.07 \mathrm{~nm}$, from the $0.1 \mathrm{~mm}$ slit width $\times 0.7 \mathrm{~nm} / \mathrm{mm}$ dispersion (for a 3600 groove $/ \mathrm{mm}$ grating).

Figure 5 shows Raman spectra for five atmospheric pressure $\mathrm{H}_{2}$-air flames at varying equivalence ratio, $\phi$. As $\phi$ decreases, $T$ increases which lowers $\rho$ (reducing the overall Raman signal strength) but also increases the population fractions of the higher rotational and vibrational levels ("spreading out" the $\mathrm{H}_{2}$ and $\mathrm{H}_{2} \mathrm{O}$ Raman signals). Figure 6 shows the $\mathrm{H}_{2}$ Raman signal for the $\phi=1.6$ flame compared to theoretical Raman spectra at three $T$ values. A manual best fit gives $2400 \mathrm{~K}$. compared to an expected adiabatic $T\left(T_{\text {cut }}\right)$ of $2220 \mathrm{~K}$. Measured $T$ s for all flames are high compared to $T_{\text {add }} \mathrm{s}: \phi=2.0 . T=2350 \mathrm{~K}, T_{u l}=2060: \phi=2.9, T=2200 \mathrm{~K}, T_{\text {cud }}=1780$; and $\phi=4.0$, $T=1900 \mathrm{~K}, T_{u t}=1560$. This could be due the noninclusion of the the $\mathrm{O}$ and $\mathrm{S}$ rotational wings around the Q-branch or a systematic error in $\phi$ measurement. 


\section{CONCLUSIONS AND FUTURE WORK}

Using the collisional broadening data of Hussong et al. (2000) the $\mathrm{H}_{2}$ Stokes Raman Q-branch is modeled and shows no significant overlap of individual ro-vibrational lines, even up to 370 atm (from 300 to $3400 \mathrm{~K}$ ). Thus a Raman temperature measurement based on spectral shape can be implemented even in high pressure advanced preburners. Atmospheric pressure $\mathrm{H}_{2}$-air flames show systematic errors in temperature measurement of $\sim 300 \mathrm{~K}$. Better spectrograph resolution would clarify the error source. A high resolution $0.5 \mathrm{~m}$ spectrograph is available for future work.

\section{REFERENCES}

Bergmann, V., and W. Stricker. 1995. Appl. Phys. B 61, 49.

Eckbreth, A. 1988. Laser Diagnostics for Combustion Temperature and Species. Abacus Press: Cambridge, MA.

Ford, A. L., and J. C. Browne. 1973. Atomic Data 5, 305.

Hussong, J., W Stricker, X Bruet, P Joubert, J Bonamy, D Robert, X Michaut, T Gabard, H Berger. 2000. Appl. Phys. B 70, 447.

Jennings, D. E., L. A. Rahn, and A. Owyoung. 1985. The Astrophys. Journ. 291, L15.

Lallemand. P., and P. Simova. 1968. J. Molecular Spectroscopy. 26, 262.

May, A. D., G. Varghese, J. C. Stryland, and H. L. Welsh. 1964. Canadian J. Physics 42, 1058.

Murray, J. R., and A. Javan. 1972. J. Molecular Spectroscopy 42, 1.

Placzek, G. 1934. in Handbuch der Radiologie, H. 6 T. 2 ed. E. Marx. Leipzig: Akademische Verlag. NTIS UCRL-Trans-526(L).

Rahn, L. A., R. L. Farrow, and G. J. Rosasco. 1991. Phys. Rev. 43, 6075.

Sinclair, P., J. Berger, X. Michaut, R. Saint-Loup, R. Chaux, H. Berger, J. Bonamy, and D. Robert. 1996. Phys. Rev. A 54, 402.

Weber, A. 1973. in The Raman Effect, Vol. 2: Applications. ed. A. Anderson. Marcel Dekker: New York.

Wehrmeyer, J. A., J. M. Cramer, R. H. Eskridge, and C. C. Dobson. 2001. J. Prop. Power 17. 27.

Yeralan, S., S. Pal, and R. J. Santoro. 2001. J. Prop. Power 17, 788. 


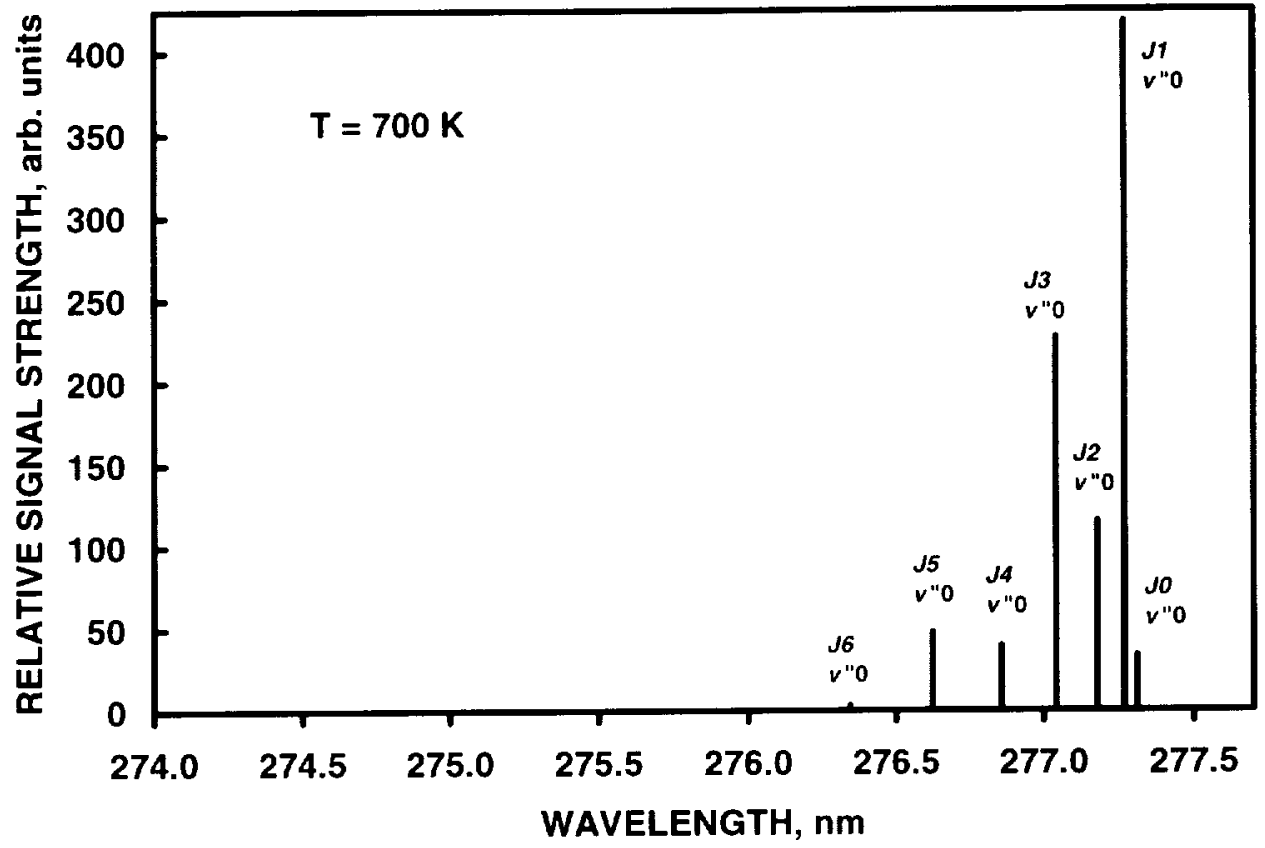

Fig. 1. $\mathrm{H}_{2}$ Stokes vibrational Q-branch Raman spectrum for $700 \mathrm{~K}$, assuming zero natural linewidth. $248.623 \mathrm{~nm}$ monochromatic laser.

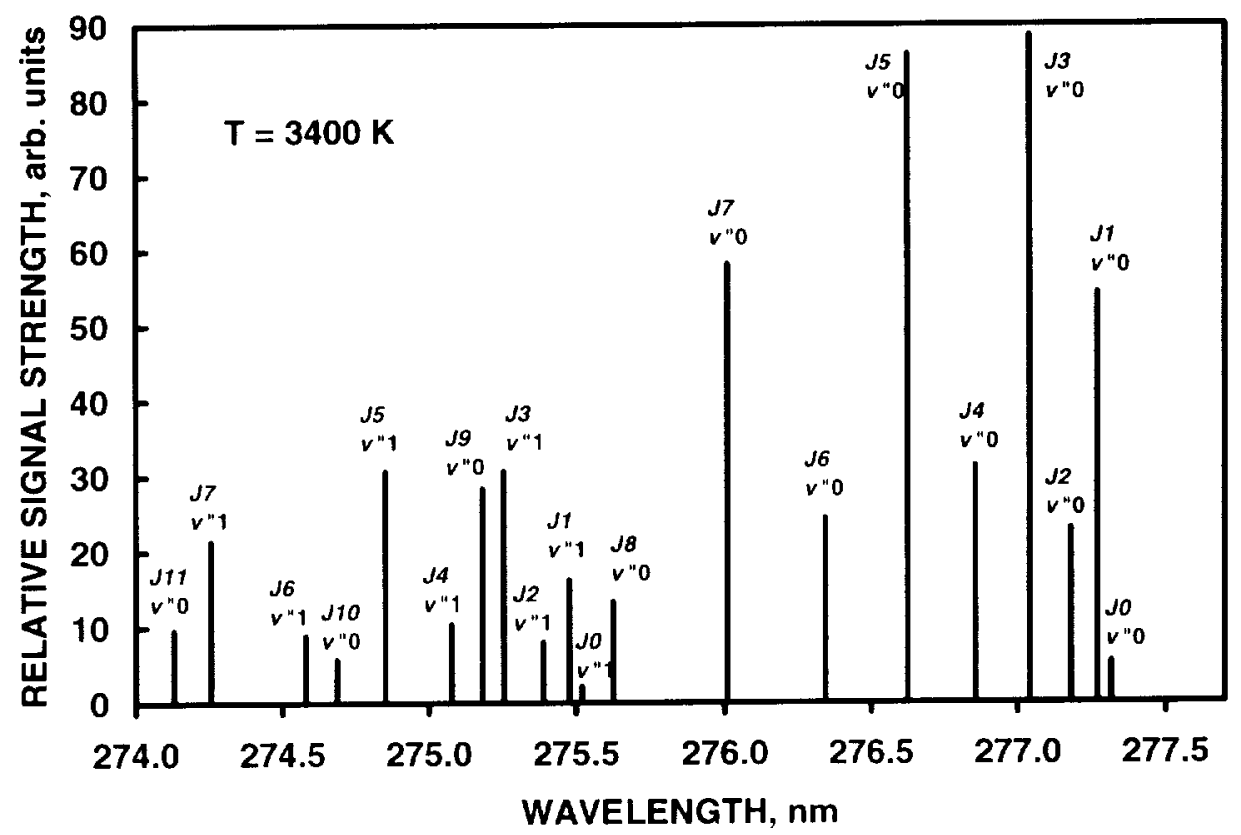

Fig. 2. $\mathrm{H}_{2}$ Stokes vibrational Q-branch Raman spectrum for $3400 \mathrm{~K}$, assuming zero natural linewidth. $248.623 \mathrm{~nm}$ monochromatic laser. 


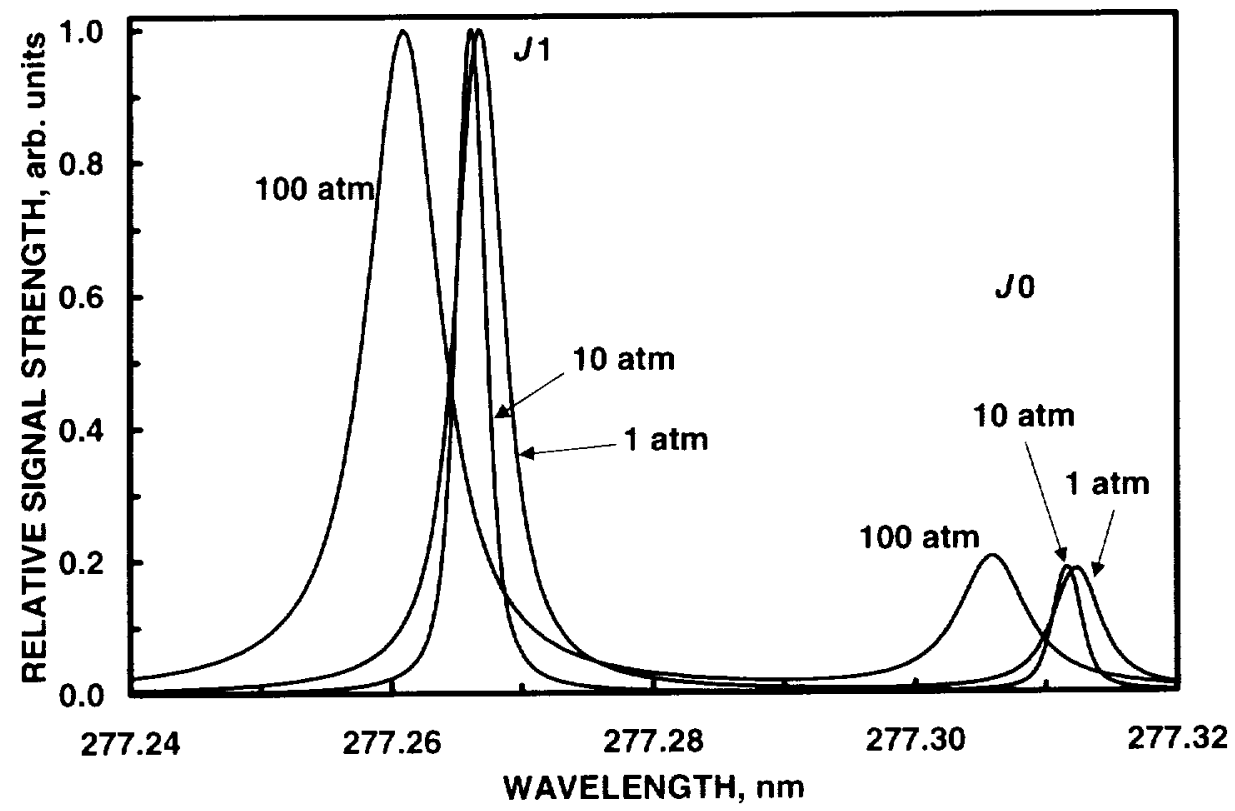

Fig. 3. First two rotational lines of $\mathrm{H}_{2}$ Stokes vibrational Q-branch spectrum for $300 \mathrm{~K}$ including natural linewidth for three pressures. Assuming dilute $\mathrm{H}_{2}$ in $\mathrm{N}_{2}$.

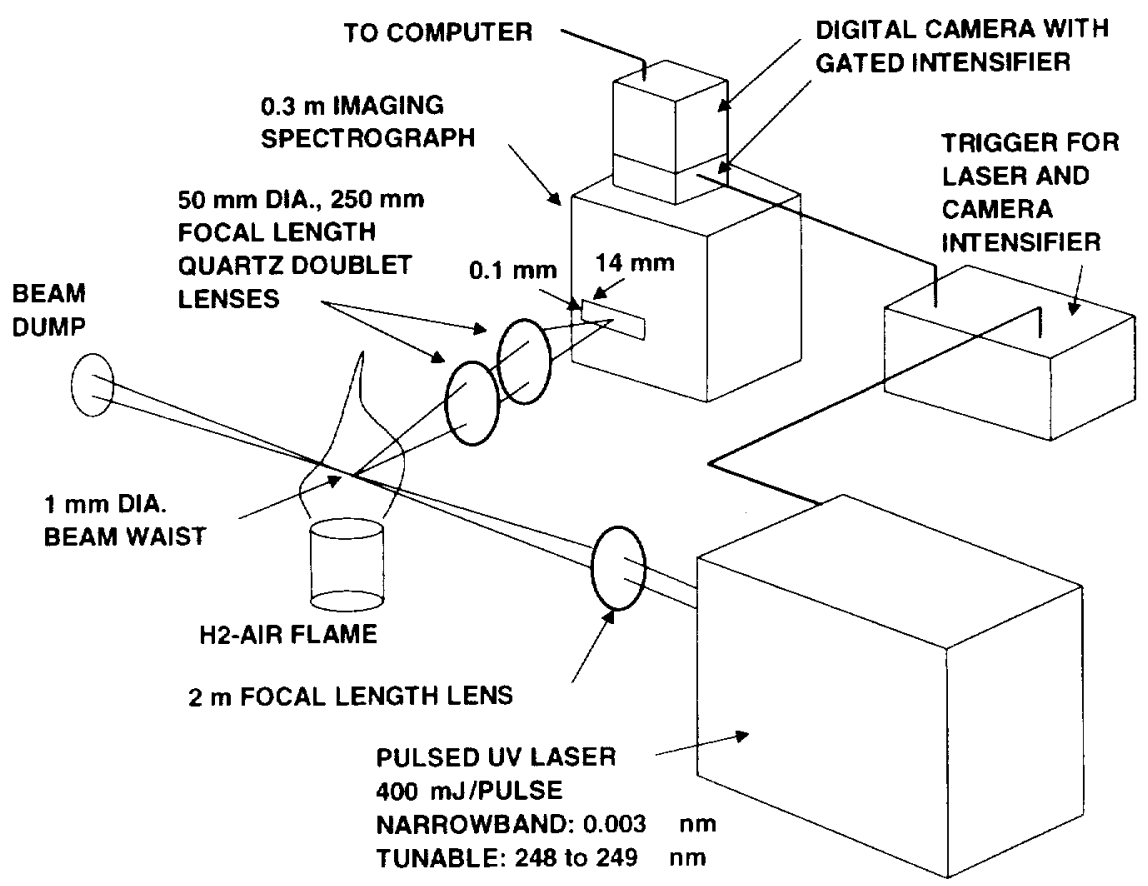

Fig. 4. Experimental system schematic. 


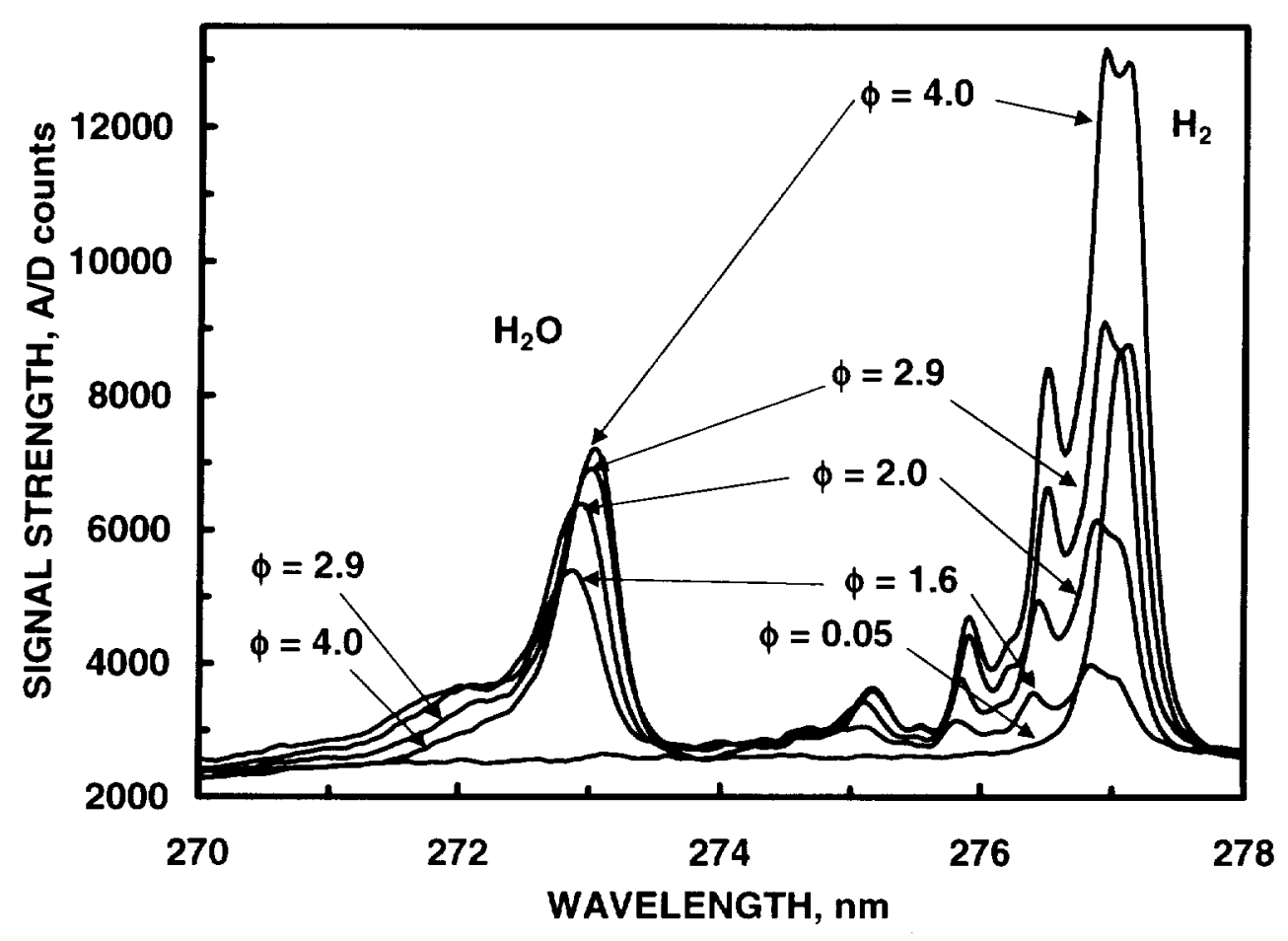

Fig. 5. Experimental Raman spectra for 1 atm $\mathrm{H}_{2}$-air flames at various equivalence ratios $(\phi)$, using $248.623 \mathrm{~nm}$ laser ( $0.003 \mathrm{~nm}$ linewidth) and imaging spectrograph $(0.2 \mathrm{~nm}$ resolution).

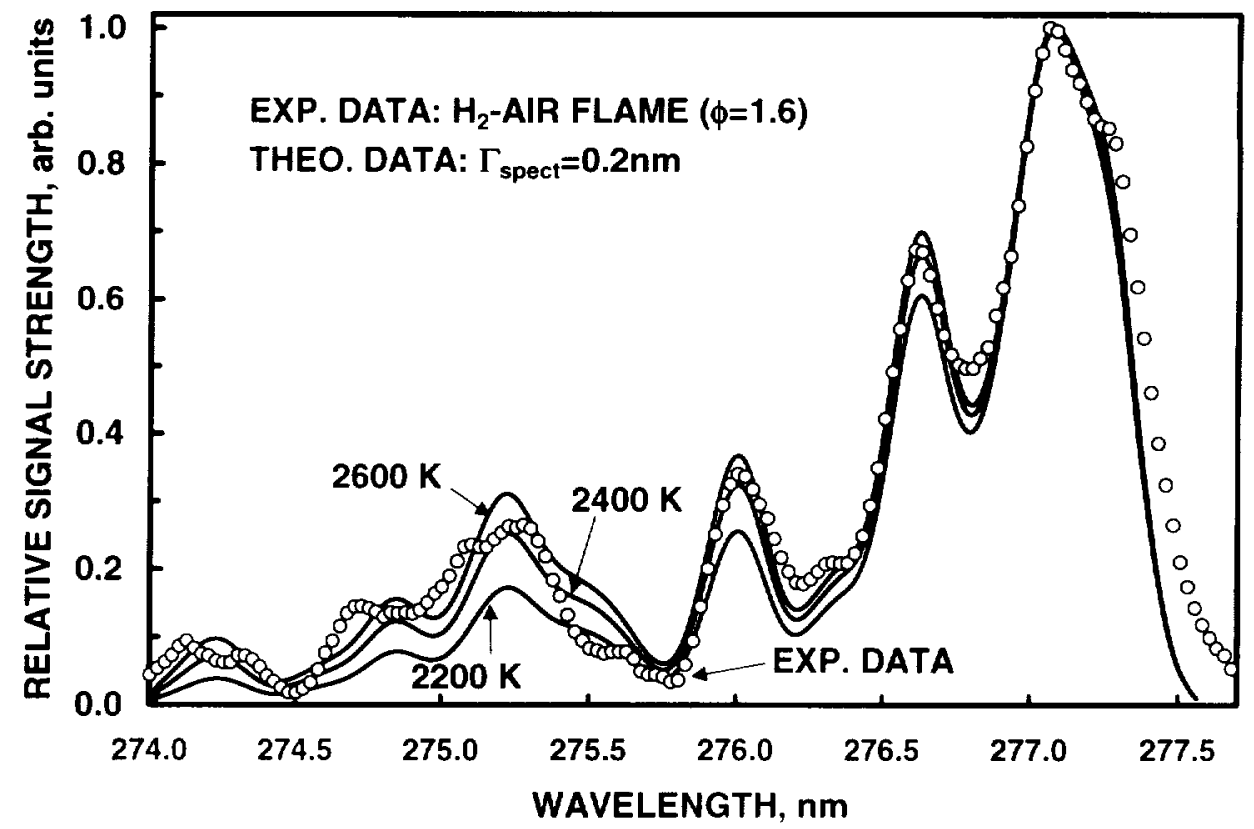

Fig. 6. Experimental $\mathrm{H}_{2}$ Stokes vibrational Q-branch Raman spectrum (for $\phi=1.6 \mathrm{H}_{2}$-air flame) compared to theoretical spectra modeled with $0.2 \mathrm{~nm}$ spectrometer resolution. 